MATHEMATICS OF COMPUTATION

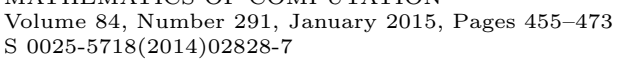

Article electronically published on March 6, 2014

\title{
APPROXIMATING AMOEBAS AND COAMOEBAS BY SUMS OF SQUARES
}

\author{
THORSTEN THEOBALD AND TIMO DE WOLFF
}

\begin{abstract}
Amoebas and coamoebas are the logarithmic images of algebraic varieties and the images of algebraic varieties under the arg-map, respectively. We present new techniques for computational problems on amoebas and coamoebas, thus establishing new connections between (co-)amoebas, semialgebraic and convex algebraic geometry and semidefinite programming.

Our approach is based on formulating the membership problem in amoebas (respectively coamoebas) as a suitable real algebraic feasibility problem. Using the real Nullstellensatz, this allows us to tackle the problem by sums of squares techniques and semidefinite programming. Our method yields polynomial identities as certificates of non-containment of a point in an amoeba or coamoeba. As the main theoretical result, we establish some degree bounds on the polynomial certificates. Moreover, we provide some actual computations of amoebas based on the sums of squares approach.
\end{abstract}

\section{INTRODUCTION}

For an ideal $I \subset \mathbb{C}\left[Z_{1}, \ldots, Z_{n}\right]$ an amoeba (introduced by Gel'fand, Kapranov, and Zelevinsky [3], see also the surveys [9] or [17]) is the image of the variety $\mathcal{V}(I)$ where each complex coordinate is mapped to (the logarithm of) its absolute value. It is often customary and useful to consider the logarithmic version of an amoeba,

$$
\mathcal{A}_{I}=\left\{\left(\log \left|z_{1}\right|, \ldots, \log \left|z_{n}\right|\right): z \in \mathcal{V}(I) \cap\left(\mathbb{C}^{*}\right)^{n}\right\}
$$

with $\mathbb{C}^{*}:=\mathbb{C} \backslash\{0\}$, and we denote the unlog amoeba by

$$
\mathcal{U}_{I}=\left\{\left(\left|z_{1}\right|, \ldots,\left|z_{n}\right|\right): z \in \mathcal{V}(I)\right\} .
$$

Similarly, the coamoeba $\mathcal{C}_{I}$ is defined as $\mathcal{C}_{I}:=\operatorname{Arg}\left(\mathcal{V}(I) \cap\left(\mathbb{C}^{*}\right)^{n}\right)$, where $\operatorname{Arg}$ denotes the mapping

$$
\left(z_{1}, \ldots, z_{n}\right) \mapsto\left(\arg \left(z_{1}\right), \ldots, \arg \left(z_{n}\right)\right) \in \mathbb{T}^{n}:=(\mathbb{R} / 2 \pi \mathbb{Z})^{n}
$$

and arg denotes the argument of a complex number (see [11 14]). If $I$ is a principal ideal generated by a polynomial $f$, for brevity we write $\mathcal{A}_{f}:=\mathcal{A}_{\langle f\rangle}$ and analogously $\mathcal{U}_{f}, \mathcal{C}_{f}$

Received by the editor April 13, 2011 and, in revised form, February 14, 2013 and April 19, 2013.

2010 Mathematics Subject Classification. Primary 14P10, 14Q10, 90C22.

Key words and phrases. Amoebas, sums of squares, real Nullstellensatz, coamoebas, semidefinite programming.

This research was supported by DFG grant TH 1333/2-1.

The first author was supported by the Alexander von Humboldt-Foundation.

(C)2014 American Mathematical Society Reverts to public domain 28 years from publication 
Studying computational questions of amoebas was initiated in [26], where in particular, certain special classes of amoebas (e.g., two-dimensional amoebas, amoebas of Grassmannians) were studied. One of the natural and fundamental computational questions is the membership problem, which asks for a given point $\left(\lambda_{1}, \ldots, \lambda_{n}\right)$ whether this point is contained in an (unlog) amoeba, respectively, coamoeba.

In 20] Purbhoo provided a characterization for the points in the complement of a hypersurface amoeba which can be used to numerically approximate the amoeba. His lopsidedness criterion provides an inequality-based certificate for non-containment of a point in an amoeba, but does not provide an algebraic certificate (in the sense of a polynomial identity certifying the non-containment). The certificates are given by iterated resultants. With this technique the amoeba can be approximated by a limit process. The computational efforts of computing the resultants are growing quite fast, and the convergence is slow.

A different approach to tackle computational problems on amoebas is to apply suitable Nullstellensätze or Positivstellensätze from real algebraic geometry or complex geometry. For some natural problems a direct approach via the Nullstellensatz (applied on a realification of the problem) is possible. Using a degree truncation approach, this allows us to find sum-of-squares-based polynomial identities which certify that a certain point is located outside of an amoeba or coamoeba. In particular, it is well known from recent lines of research in computational semialgebraic geometry (see, e.g., [4, 5, 15]) that these certificates can be computed via semidefinite programming (SDP).

In this paper, we discuss theoretical foundations as well as some practical issues of such an approach, thus establishing new connections between amoebas, semialgebraic and convex algebraic geometry and semidefinite programming. First, we present various Nullstellensatz-type formulations (a standard approach in Corollary 3.2 and a monomial approach in Corollary [3.7) and compare their properties to a recent toric Nullstellensatz of Niculescu and Putinar [10]. Using a degree truncation approach this yields a sequence of supersets of the amoeba $\mathcal{A}_{f}$, which converges to $\mathcal{A}_{f}$ (Theorem 3.11). For every fixed superset in this sequence, the membership problem can be solved by semidefinite programming.

The main theoretical contribution is contained in Section 4. For one of our approaches, we can provide some degree bounds for the certificates (Corollary 4.5). It is remarkable and even somewhat surprising that these degree bounds are derived from Purbhoo's lopsidedness criterion (which is not at all sum-of-squares-based). We also show that in certain cases (such as for the Grassmannian of lines) the degree bounds can be reduced to simpler amoebas (Theorem 4.7).

In Section 5 we provide some actual computations on this symbolic-numerical approach. Besides providing some results on the membership problem itself, we will also consider more sophisticated versions (such as bounding the diameter of a complement component for certain classes).

Finally, in Section [6 we give an outlook on further questions on the approach initiated in the current paper.

\section{Preliminaries}

In the following, let $\mathbb{C}[Z]=\mathbb{C}\left[Z_{1}, \ldots, Z_{n}\right]$ denote the polynomial ring over $\mathbb{C}$ in $n$ variables. For $f=\sum_{\alpha \in A} c_{\alpha} Z^{\alpha} \in \mathbb{C}[Z]$, the Newton polytope $\operatorname{New}(f)=\operatorname{conv}\{\alpha \in$ 


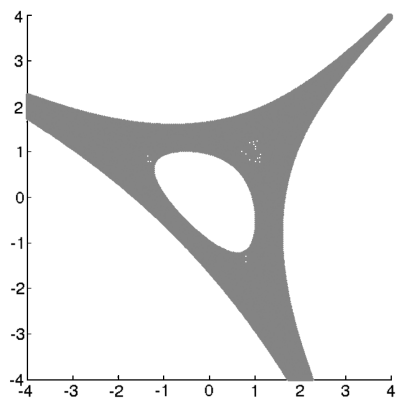

Figure 1 . The amoeba of $f=Z_{1}^{2} Z_{2}+Z_{1} Z_{2}^{2}-4 Z_{1} Z_{2}+1$.
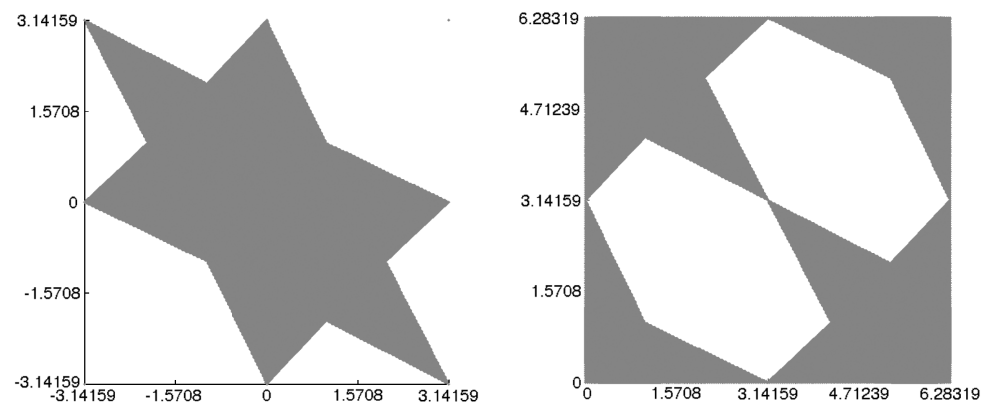

Figure 2. The coamoeba of $f=Z_{1}^{2} Z_{2}+Z_{1} Z_{2}^{2}-4 Z_{1} Z_{2}+1$ in two different views of $\mathbb{T}^{2}$, namely $[-\pi, \pi)^{2}$ versus $[0,2 \pi)^{2}$.

$\left.\mathbb{N}_{0}^{n}: \alpha \in \operatorname{supp}(f)\right\}$ of $f$ is the convex hull of the exponent vectors, where $\operatorname{supp}(f)$ denotes the support of $f$.

2.1. Amoebas and coamoebas. We recall some basic statements about amoebas (see $2,2,17]$ ). For any ideal $I \subset \mathbb{C}[Z]$, the amoeba $\mathcal{A}_{I}$ is a closed set. For a polynomial $f \in \mathbb{C}[Z]$, the complement of the hypersurface amoeba $\mathcal{A}_{f}$ consists of finitely many convex regions, and these regions are in bijective correspondence with the different Laurent expansions of the rational function $1 / f$. See Figure 1 for an example.

The order $\nu$ of a point $w$ in the complement $\mathbb{R}^{n} \backslash \mathcal{A}_{f}$ is given by

$$
\nu_{j}=\frac{1}{(2 \pi i)^{n}} \int_{\log ^{-1}(w)} \frac{z_{j} \partial_{j} f(z)}{f(z)} \frac{d z_{1} \cdots d z_{n}}{z_{1} \cdots z_{n}}, 1 \leq j \leq n,
$$

where $\log (z)$ is defined as $\log (z)=\left(\log \left|z_{1}\right|, \ldots, \log \left|z_{n}\right|\right)$. The order mapping induces an injective map from the set of complement components into New $(f) \cap \mathbb{Z}^{n}$. The complement components corresponding to the vertices of New $(f)$ do always exist [2].

Similarly, for $f \in \mathbb{C}[Z]$ any connected component of the coamoeba complement $\mathbb{T}^{n} \backslash \mathcal{C}_{f}$ is a convex set (see Figure 2). If $\overline{\mathcal{C}_{f}}$ denotes the closure of $\mathcal{C}_{f}$ in the torus $\mathbb{T}^{n}$, then the number of connected components of $\mathbb{T}^{n} \backslash \overline{\mathcal{C}_{f}}$ is bounded by $n$ ! vol New $(f)$ (Nisse [14, Thm. 5.19]), where vol denotes the volume. 
For technical reasons (see Theorem 3.6 ) it will be often convenient to consider in the definition of a coamoeba also those points $z \in \mathcal{V}(I)$ which have a zerocomponent. Namely, if a zero $z$ of $I$ has a zero-component $z_{j}=0$, then we associate this component to any phase. Call this modified version of a coamoeba $\mathcal{C}_{I}^{\prime}$, i.e.,

$$
\mathcal{C}_{I}^{\prime}:=\left\{\phi \in \mathbb{T}^{n}: \exists z \in \mathcal{V}(I): \arg \left(z_{j}\right)=\phi_{j} \text { or } z_{j}=0 \text { for } 1 \leq j \leq n\right\} .
$$

Note that for principal ideals $I=\langle f\rangle$ the difference between $\mathcal{C}_{I}$ and $\mathcal{C}_{I}^{\prime}$ solely may occur at points which are contained in the closure of $\mathcal{C}_{I}$. The set-theoretic difference of $\mathcal{C}_{I}$ and $\mathcal{C}_{I}^{\prime}$ is a lower-dimensional subset of $\mathbb{R}^{n}$ (since in each environment of a point in $\mathcal{C}_{I}^{\prime} \backslash \mathcal{C}_{I}$ we have a coamoeba point).

2.2. The situation at $\infty$. It is well known that the geometry of amoebas at infinity (i.e., the "tentacles") can be characterized in terms of logarithmic limit sets and tropical geometry, and thus amoebas form one of the building blocks of tropical geometry (for general background on tropical geometry we refer to [8, 9,22 ).

For (large) $R>0$ let $\mathcal{A}^{(R)}$ denote the scaled version $\mathcal{A}_{I}^{(R)}:=\frac{1}{R} \mathcal{A}_{I} \cap \mathbb{S}^{n-1}$, where $\mathbb{S}^{n-1}$ denotes the $(n-1)$-dimensional unit sphere. Extending this definition, the logarithmic limit set $\mathcal{A}_{I}^{(\infty)}$ is the set of points $v \in \mathbb{S}^{n-1}$ such that there exists a sequence $v_{R} \in \mathcal{A}_{I}^{(R)}$ with $\lim _{R \rightarrow \infty} v_{R}=v$. For a polynomial $f=\sum_{\alpha} c_{\alpha} Z^{\alpha} \in \mathbb{C}[Z]$ denote by trop $f:=\bigoplus 0 \odot Z_{1}^{\alpha_{1}} \odot \cdots \odot Z_{n}^{\alpha_{n}}$ its tropicalization (with respect to the trivial valuation) over the tropical semiring $(R, \oplus, \odot):=(\mathbb{R} \cup\{-\infty\}$, max, +$)$. Then (see [8,24]):

Proposition 2.1. A vector $w \in \mathbb{R}^{n} \backslash\{0\}$ is contained in the tropical variety of $I$ if and only if the corresponding unit vector $\frac{1}{\|w\|} w$ is contained in $\mathcal{A}_{I}^{(\infty)}$. Thus the tropical variety of $I$ coincides with the cone over the logarithmic limit set $\mathcal{A}_{I}^{(\infty)}$.

\section{Approximations Based on the ReAl Nullstellensatz}

We study certificates of points in the complement of the amoeba based on the real Nullstellensatz and compare them to existing statements in the literature (such as the toric Nullstellensatz of Niculescu and Putinar). By imposing degree truncations this will then yield a hierarchy of certificates of bounded degree.

We use the following real Nullstellensatz (see, e.g., [1,19]):

Proposition 3.1. For polynomials $g_{1}, \ldots, g_{r} \in \mathbb{R}[X]$ and $I:=\left\langle g_{1}, \ldots, g_{r}\right\rangle \subset \mathbb{R}[X]$ the following statements are equivalent:

- The real variety $\mathcal{V}_{\mathbb{R}}(I)$ is empty.

- There exist a polynomial $G \in I$ and a sum of squares polynomial $H$ with

$$
G+H+1=0 \text {. }
$$

Given $\lambda \in(0, \infty)^{n}$, the question if $\lambda$ is contained in the unlog amoeba $\mathcal{U}_{I}$ can be phrased as the real solvability of a real system of polynomial equations. For a polynomial $f \in \mathbb{C}[Z]=\mathbb{C}\left[Z_{1}, \ldots, Z_{n}\right]$ let $f^{\text {re }}, f^{\text {im }} \in \mathbb{R}[X, Y]=\mathbb{R}\left[X_{1}, \ldots, X_{n}, Y_{1}, \ldots, Y_{n}\right]$ be its real and imaginary parts, i.e.,

$$
f(Z)=f(X+i Y)=f^{\mathrm{re}}(X, Y)+i \cdot f^{\mathrm{im}}(X, Y) .
$$

We consider the ideal $I^{\prime} \subset \mathbb{R}[X, Y]$ generated by the polynomials

$$
\left\{f_{j}^{\mathrm{re}}, f_{j}^{\mathrm{im}}: 1 \leq j \leq r\right\} \cup\left\{X_{k}^{2}+Y_{k}^{2}-\lambda_{k}^{2}: 1 \leq k \leq n\right\} .
$$


Corollary 3.2. Let $I=\left\langle f_{1}, \ldots, f_{r}\right\rangle$, and $\lambda \in(0, \infty)^{n}$. Either the point $\lambda$ is contained in $\mathcal{U}_{I}$, or there exist a polynomial $G \in I^{\prime} \subset \mathbb{R}[X, Y]$ and a sum of squares polynomial $H \in \mathbb{R}[X, Y]$ with

$$
G+H+1=0 .
$$

Proof. For any polynomial $f \in \mathbb{C}[Z]$ it suffices to observe that a point $z=x+i y$ is contained in $\mathcal{V}(f)$ if and only if $(x, y) \in \mathcal{V}_{\mathbb{R}}\left(f^{\mathrm{re}}\right) \cap \mathcal{V}_{\mathbb{R}}\left(f^{\mathrm{im}}\right)$, and that $\left|z_{k}\right|=$ $\lambda_{k}$ if and only if $(x, y) \in \mathcal{V}_{\mathbb{R}}\left(X^{2}+Y^{2}-\lambda_{k}^{2}\right)$. Then the statement follows from Proposition 3.1

Corollary 3.2 states that for any point $\lambda \notin \mathcal{U}_{I}$ there exists a certificate

$$
\sum_{j=1}^{r} p_{j} f_{j}^{\mathrm{re}}+\sum_{j=1}^{r} p_{j}^{\prime} f_{j}^{\mathrm{im}}+\sum_{k=1}^{n} q_{k}\left(X_{k}^{2}+Y_{k}^{2}-\lambda_{k}^{2}\right)+H+1=0
$$

with polynomials $p_{j}, p_{j}^{\prime}, q_{k}$ and a sum of squares $H$. We refer to these certificates as certificates in the standard approach.

We say that a certificate of the form (3.3) is of degree at most $d$ if the (total) degree of each summand in (3.3) is at most $d$.

Remark 3.3. By the following fact (which is easy to check), the sum of squares condition (3.2) can also be stated as

-1 is a sum of squares in the quotient ring $\mathbb{R}[X, Y] / I^{\prime}$.

Fact 3.4 (Parrilo [16]). Let $I=\left\langle g_{1}, \ldots, g_{r}\right\rangle \subset \mathbb{R}[X]$ and $f \in \mathbb{R}[X]$. There exist $p_{1}, \ldots, p_{r} \in \mathbb{R}[X]$ such that

$$
f+\sum_{i} p_{i} g_{i} \text { is a sum of squares in } \mathbb{R}[X]
$$

if and only if $f$ is a sum of squares in $\mathbb{R}[X] / I$.

Any two of these equivalent conditions in Fact 3.4 is a certificate for the nonnegativity of $f$ on the variety $I$.

Before stating a coamoeba version, we note the following normalization properties. Whenever it is needed for amoebas, we can assume that the point $\lambda$ in the amoeba membership problem is the all-1-vector $\mathbf{1} \in \mathbb{R}^{n}$. Similarly, for the coamoeba membership problem we can assume that the investigated point is the origin $0 \in \mathbb{T}^{n}$.

Lemma 3.5. Let $I=\left\langle f_{1}, \ldots, f_{r}\right\rangle$.

(1) A point $\left(\lambda_{1}, \ldots, \lambda_{n}\right) \in(0, \infty)^{n}$ is contained in $\mathcal{U}_{I}$ if and only if $\mathbf{1} \in$ $\mathcal{U}_{\left\langle g_{1}, \ldots, g_{r}\right\rangle}$, where

$$
g_{j}\left(Z_{1}, \ldots, Z_{n}\right):=f_{j}\left(\lambda_{1} Z_{1}, \ldots, \lambda_{n} Z_{n}\right), \quad 1 \leq j \leq r .
$$

(2) A point $\left(z_{1}, \ldots, z_{n}\right)$ is contained in $\mathcal{V}(I)$ with $\arg z_{j}=\mu_{j}$ if and only if the (non-negative) real vector $y$ with $y_{j}:=z_{j} e^{-i \mu_{j}}$ is contained in $\mathcal{V}\left(g_{1}, \ldots, g_{r}\right)$ where

$$
g_{j}\left(Z_{1}, \ldots, Z_{n}\right):=f_{j}\left(Z_{1} e^{i \mu_{1}}, \ldots, Z_{n} e^{i \mu_{n}}\right), \quad 1 \leq j \leq r .
$$

Proof. A point $\left(z_{1}, \ldots, z_{n}\right)$ is contained in $\mathcal{V}(I)$ with $\left|z_{j}\right|=\lambda_{j}$ if and only if the vector $y$ defined by $y_{j}:=z_{j} / \lambda_{j}$ is contained in $\mathcal{V}\left(g_{1}, \ldots, g_{r}\right)$ with $\left|y_{j}\right|=1$. The second statement follows similarly. 
Theorem 3.6. Let $I=\left\langle f_{1}, \ldots, f_{r}\right\rangle$. The point $(0, \ldots, 0)$ is contained in the complement of the coamoeba $\mathcal{C}_{I}^{\prime}$ if and only if there exists a polynomial identity

$$
\sum_{j=1}^{r} c_{j} \cdot f_{j}\left(X^{2}, Y\right)^{\mathrm{re}}+\sum_{j=1}^{r} c_{j}^{\prime} \cdot f_{j}\left(X^{2}, Y\right)^{\mathrm{im}}+\sum_{k=1}^{n} d_{k} \cdot Y_{k}+H+1=0
$$

with polynomials $c_{j}, c_{j}^{\prime}, d_{k} \in \mathbb{R}[X, Y]$ and a sum of squares $H$. Here, $f_{j}\left(X^{2}, Y\right)$ abbreviates $f_{j}\left(X_{1}^{2}, \ldots, X_{n}^{2}, Y_{1}, \ldots, Y_{n}\right)$.

Proof. Note that the statement $0 \in \mathcal{C}_{I}^{\prime}$ is equivalent to $\left\{z=x+i y \in \mathbb{C}^{n}: z \in\right.$ $\mathcal{V}(I)$ and $\left.x_{k} \geq 0, y_{k}=0,1 \leq k \leq n\right\} \neq \emptyset$. Moreover, observe that the condition $x_{k} \geq 0$ can be replaced by considering $X_{k}^{2}$ in the arguments of $f_{1}, \ldots, f_{r}$. Hence, by Proposition 3.1 the statement $0 \notin \mathcal{C}_{I}^{\prime}$ is equivalent to the existence of a polynomial identity of the form (3.4).

Observe that in the proof the use of $\mathcal{C}_{I}^{\prime}$ (rather than $\mathcal{C}_{I}$ ) allowed us to use the basic Nullstellensatz (rather than a Positivstellensatz, which would have introduced several sum of squares polynomials).

The following variant of the Nullstellensatz approach will allow us to obtain degree bounds (see Section (4). For vectors $\alpha(1), \ldots, \alpha(d) \in \mathbb{N}_{0}^{n}$ and coefficients $b_{1}, \ldots, b_{d} \in \mathbb{C}^{*}$ let $f=\sum_{j=1}^{d} b_{j} \cdot Z^{\alpha(j)} \in \mathbb{C}[Z]$. For any given values of $\lambda_{1}, \ldots, \lambda_{n}$ set

$$
\mu_{j}:=\lambda^{\alpha(j)}=\lambda_{1}^{\alpha(j)_{1}} \cdots \lambda_{n}^{\alpha(j)_{n}}, \quad 1 \leq j \leq d .
$$

If the rank of the matrix with columns $\alpha(1), \ldots, \alpha(d)$ is $n$ (i.e., the vectors $\alpha(1), \ldots, \alpha(d)$ span $\left.\mathbb{R}^{n}\right)$, then the $\lambda$-values can be reconstructed uniquely from the $\mu$-values. We come up with the following variant of a Nullstellensatz.

Here, let $I:=\left\langle f_{1}, \ldots, f_{r}\right\rangle$ such that $f_{i}$ is of the form $\sum_{j=1}^{d_{i}} b_{i j} Z^{\alpha(i, j)}$ with $\alpha(i, j) \in$ $\mathbb{N}_{0}^{n}$. Let $m_{i j}$ be the monomial $m_{i j}=Z^{\alpha(i, j)}=Z_{1}^{\alpha(i, j)_{1}} \cdots Z_{n}^{\alpha(i, j)_{n}}$. We consider the ideal $I^{*} \subset \mathbb{R}[X, Y]$ generated by the polynomials

$$
\left\{f_{i}^{\mathrm{re}}, f_{i}^{\mathrm{im}}: 1 \leq i \leq r\right\} \cup\left\{\left(m_{i j}^{\mathrm{re}}\right)^{2}+\left(m_{i j}^{\mathrm{im}}\right)^{2}-\mu_{i j}^{2}: 1 \leq j \leq d_{i}, 1 \leq i \leq r\right\},
$$

where $\mu_{i j}=\lambda^{\alpha(i, j)}$.

Corollary 3.7. Let $I:=\left\langle f_{1}, \ldots, f_{r}\right\rangle$, and assume that the set $\bigcup_{i=1}^{r} \bigcup_{j=1}^{d_{i}}\{\alpha(i, j)\}$ spans $\mathbb{R}^{n}$. Either a point $\lambda \in(0, \infty)^{n}$ is contained in $\mathcal{U}_{I}$, or there exist polynomials $G \in I^{*} \subset \mathbb{R}[X, Y]$ and a sum of squares polynomial $H \in \mathbb{R}[X, Y]$ with

$$
G+H+1=0 .
$$

We refer to these certificates as certificates in the monomial approach.

For hypersurface amoebas of real polynomials, the membership problem relates to the following statement of Niculescu and Putinar [10. Let $p=p(X, Y) \in$ $\mathbb{R}\left[X_{1}, \ldots, X_{n}, Y_{1}, \ldots, Y_{n}\right]$ be a real polynomial. Then $p$ can be written as a complex polynomial $p(X, Y)=P(Z, \bar{Z})$ with $P \in \mathbb{C}\left[Z_{1} \ldots, Z_{n}, \bar{Z}_{1}, \ldots, \bar{Z}_{n}\right]$ and $\overline{P(Z, \bar{Z})}=$ $P(Z, \bar{Z})$. Note that there exists a polynomial $Q \in \mathbb{C}\left[Z_{1}, \ldots, Z_{n}\right]$ with

$$
p(x, y)^{2}=|P(z, \bar{z})|^{2}=|Q(z)|^{2} \text { for } z \in T^{n},
$$

where $T:=\{z \in \mathbb{C}:|z|=1\}$. 
The following statement can be obtained by applying the Nullstellensatz on the set $\left\{z=(x, y):|q(z)|^{2}=1,\left|z_{1}\right|^{2}=1, \ldots,\left|z_{n}\right|^{2}=1\right\}$, then applying Putinar's Theorem [21] on the multiplier polynomial of $|q(Z)|^{2}$ (see [10]).

Proposition 3.8. Let $q \in \mathbb{C}\left[Z_{1}, \ldots, Z_{n}\right]$. Then $q(z) \neq 0$ for all $z \in T^{n}$ if and only if there are complex polynomials $p_{1}, \ldots, p_{k}, r_{1}, \ldots, r_{l} \in \mathbb{C}\left[Z_{1}, \ldots, Z_{n}\right]$ with

$$
1+\left|p_{1}(z)\right|^{2}+\cdots+\left|p_{k}(z)\right|^{2}=|q(z)|^{2}\left(\left|r_{1}(z)\right|^{2}+\cdots+\left|r_{l}(z)\right|^{2}\right), \quad z \in T^{n}
$$

Note that the statement is not an identity of polynomials, but an identity for all $z$ in the $n$-torus $T^{n}$.

While Proposition 3.8 provides a nice structural result, due to the following reasons we prefer Corollary 3.2 for actual computations. In representation (3.7), two sums of squares polynomials (rather than just one as in (3.2)) are needed in the representation, and the degree is increased (by the squaring process). Moreover, the theorem is not really a representation theorem (in terms of an identity of polynomials), but an identity over $T^{n}$; therefore in order to express this computationally, the polynomials hidden in this equivalence (i.e., the polynomials $1-\left|Z_{1}\right|^{2}, \ldots, 1-\left|Z_{n}\right|^{2}$ ) have to be additionally used.

SOS-based approximations. By putting degree truncations on the certificates, we can transform the theoretic statements into effective algorithmic procedures for constructing certificates. The idea of degree truncations in polynomial identities follows the same principles of the degree truncations with various types of Nullstellensätze and Positivstellensätze in [4,6, 15. It is instructive to have a look at two simple examples first.

Example 3.9. Let $f$ be the polynomial $f=Z+z_{0}$ with a complex constant $z_{0}=x_{0}+i y_{0}$. The ideal $I^{\prime}$ of interest is defined by

$$
\begin{aligned}
h_{1} & :=f^{\mathrm{re}}=X+x_{0}, \\
h_{2} & :=f^{\mathrm{im}}=Y+y_{0}, \\
h_{3} & :=X^{2}+Y^{2}-\lambda^{2} .
\end{aligned}
$$

For values of $\lambda \geq 0$ which correspond to points outside the amoeba (i.e., $\lambda^{2} \neq x_{0}^{2}+$ $\left.y_{0}^{2}\right)$, we have $\mathcal{V}_{\mathbb{C}}(I)=\emptyset$ and thus the Gröbner basis $G$ of $\left\langle h_{1}, h_{2}, h_{3}\right\rangle$ is $G=\{1\}$. The corresponding multiplier polynomials $p_{j}$ that represent 1 as a linear combination $\sum_{j} p_{j} h_{j}$ are

$$
p_{1}=\frac{-X+x_{0}}{x_{0}^{2}+y_{0}^{2}-\lambda^{2}}, \quad p_{2}=\frac{-Y+y_{0}}{x_{0}^{2}+y_{0}^{2}-\lambda^{2}}, \quad p_{3}=\frac{1}{x_{0}^{2}+y_{0}^{2}-\lambda^{2}} .
$$

Hence, in particular, -1 can be written as a sum of squares in the quotient ring $\mathbb{R}[X] / I^{\prime}$. The necessary degree with regard to equation (3.2) is just 2 .

For $\lambda^{2}=x_{0}^{2}+y_{0}^{2}$, the Gröbner basis (w.r.t. a lexicographic variable ordering with $X \succ Y)$ is

$$
X+x_{0}, Y+y_{0}
$$

The point $\left(-x_{0},-y_{0}\right)$ is contained in $\mathcal{V}_{\mathbb{R}}\left(I^{\prime}\right)$; thus in this case there does not exist a Nullstellensatz-type certificate.

Example 3.10. Consider the polynomial $f=Z_{1}+Z_{2}+5$ with $Z_{j}=X_{j}+i Y_{j}$. The ideal $I^{\prime}$ of interest is defined by $h_{1}:=X_{1}+X_{2}+5, h_{2}:=Y_{1}+Y_{2}, h_{3}:=X_{1}^{2}+Y_{1}^{2}-\lambda_{1}^{2}, h_{4}:=X_{2}^{2}+Y_{2}^{2}-\lambda_{2}^{2}$. 
Consider $\lambda_{1}=2, \lambda_{2}=3$. Using a lexicographic ordering with $X_{1} \succ X_{2} \succ Y_{1} \succ Y_{2}$, a Gröbner basis is

$$
Y_{2}^{2}, Y_{1}+Y_{2}, X_{2}+3, X_{1}+2 .
$$

The standard monomials are 1 and $Y_{2}$. It is easy to see that -1 is not a sum of squares in the quotient ring, which reflects the fact that $(2,3) \in \mathcal{U}_{I}$.

Consider now the choice $\lambda_{1}=1$ and $\lambda_{2}=2$. Using lexicographic ordering again, the Gröbner basis is

$$
25 Y_{2}^{2}+96, Y_{1}+Y_{2}, 5 X_{2}+14,5 X_{1}+11 .
$$

Hence, $25 Y_{2}^{2} \equiv-96 \bmod I^{\prime}$, which gives the sum of squares identity

$$
\left(\frac{5}{\sqrt{96}} Y_{2}\right)^{2} \equiv-1 \bmod I^{\prime}
$$

and thus shows $(1,2) \notin \mathcal{U}_{I}$.

Using the degree truncation approach for sums of squares we can for a given ideal $I=\left\langle f_{1}, \ldots, f_{r}\right\rangle$ define

$$
\begin{aligned}
C_{t}:=\left\{\lambda \in(0, \infty)^{n} \backslash \mathcal{U}_{I}:\right. & \text { there exists a certificate of the form (3.3) } \\
& \text { for } \lambda \text { of degree } \leq 2 t\} .
\end{aligned}
$$

Similarly, for coamoebas $\mathcal{C}_{I}$ let $D_{t}$ be the subsets of its complement obtained by the degree truncation. These sequences are the basis of the effective implementation (see Section 5).

Theorem 3.11. Let $I=\left\langle f_{1}, \ldots, f_{r}\right\rangle$ and $t_{0}:=\max _{j}\left\lceil\operatorname{deg} f_{j} / 2\right\rceil$. The sequence $\left(C_{t}\right)_{t \geq t_{0}}$ converges pointwise to the complement of the unlog amoeba $\mathcal{U}_{I}$, and it is monotone increasing in the set-theoretic sense, i.e., $C_{t} \subset C_{t+1}$ for $t \geq t_{0}$.

Similarly, the sequence $\left(D_{t}\right)_{t \geq t_{0}}$ converges pointwise to the complement of the coamoeba $\mathcal{C}_{I}$, and it is monotone increasing in the set-theoretic sense, i.e., $D_{t} \subset$ $D_{t+1}$ for $t \geq t_{0}$.

Proof. For any given point $z$ in the complement of the amoeba there exists a certificate of minimal degree, say $d$. For $t<\lceil d / 2\rceil$ the point $z$ is not contained in $C_{t}$ and for $t \geq\lceil d / 2\rceil$ the point $z$ is contained in $C_{t}$. In particular, the relaxation process is monotone increasing. And analogously for coamoebas.

Remark 3.12. A similar result holds for the monomial approach. Namely, for a given ideal $I=\left\langle f_{1}, \ldots, f_{r}\right\rangle, t_{0}:=\max _{j}\left\lceil\operatorname{deg} f_{j} / 2\right\rceil$, and $I^{*}$ generated by the polynomials (3.5), the sets

$C_{t}^{*}:=\left\{\lambda \in(0, \infty)^{n} \backslash \mathcal{U}_{I} \quad: \quad\right.$ there exists a certificate of the form $G+H+1=0$ with $G \in I^{*}$ and $H$ SOS for $\lambda$ of degree $\left.\leq 2 t\right\}$,

$\left(t \geq t_{0}\right)$ converge pointwise to the complement of $\mathcal{U}_{I}$ and, set-theoretically, this sequence is monotone increasing.

It is well known (and at the heart of current developments in optimization of polynomial functions, see [4, 15] or the survey [5]) that SOS conditions with degree constraints of the form (3.3) can be phrased as semidefinite programs. Finding an (optimal) positive semidefinite matrix within an affine linear variety is known as semidefinite programming (see e.g. 28] for a comprehensive treatment). Semidefinite programs can be solved efficiently both in theory and in practice. 
Precisely, any sum of squares polynomial $H$ can be expressed as $M Q M^{T}$, where $Q$ is a symmetric positive semidefinite matrix (abbreviated $Q \succeq 0$ ) and $M$ is a vector of monomials.

Similarly, by the degree restriction the linear combination in (3.2) or (3.6) can be integrated into the semidefinite formulation by a comparison of coefficients.

\section{Special Certificates}

For a certain class of amoebas, we can provide some explicit classes of Nullstellensatz-type certificates. As a first warm-up example, we illustrate some ideas for constructing special certificates systematically for linear amoebas in the standard approach. Then we show how to construct special certificates for the monomial-based approach.

In this section we concentrate on the case of hypersurface amoebas.

Linear amoebas in the standard approach. Let $f=a Z_{1}+b Z_{2}+c$ be a general linear polynomial in two variables with real coefficients $a, b, c \in \mathbb{R}$. We consider certificates of the form (3.2) based on the third binomial formula $(\alpha+\beta)(\alpha-\beta)=$ $\alpha^{2}-\beta^{2}$, and we use the sums of squares $\left(X_{1}-X_{2}\right)^{2}$ and $\left(Y_{1}-Y_{2}\right)^{2}$. For simplicity assume $a, b>0$. Setting

$$
\begin{aligned}
G_{1}:= & \left(a X_{1}+b X_{2}-c\right)\left(a X_{1}+b X_{2}+c\right)+\left(a Y_{1}+b Y_{2}\right)\left(a Y_{1}+b Y_{2}\right) \\
& -\left(a^{2}+a b\right)\left(X_{1}^{2}+Y_{1}^{2}-\lambda_{1}^{2}\right)-\left(b^{2}+a b\right)\left(X_{2}^{2}+Y_{2}^{2}-\lambda_{2}^{2}\right), \\
H_{1}:= & a b\left(X_{1}-X_{2}\right)^{2}+a b\left(Y_{1}-Y_{2}\right)^{2},
\end{aligned}
$$

the sum $G_{1}+H_{1}$ simplifies via elementary cancellation to

$$
\gamma_{1}:=\left(a^{2}+a b\right) \lambda_{1}^{2}+\left(b^{2}+a b\right) \lambda_{2}^{2}-c^{2} .
$$

Assume that the point $\left(\lambda_{1}, \lambda_{2}\right)$ is not contained in the unlog amoeba $\mathcal{U}_{f}$. In order to obtain the desired polynomial identity (3.2) certifying containment in the complement of $\mathcal{U}_{f}$, we require $\gamma_{1}$ to be negative. In that case we have

$$
\frac{1}{\left|\gamma_{1}\right|}\left(G_{1}+H_{1}\right)+1=0
$$

which gives the polynomial identity (3.2). Analogously, we obtain for

$$
\begin{aligned}
G_{2}:= & \left(-a X_{1}+b X_{2}+c\right)\left(a X_{1}+b X_{2}+c\right)+\left(-a Y_{1}+b Y_{2}\right)\left(a Y_{1}+b Y_{2}\right) \\
& a^{2}\left(X_{1}^{2}+Y_{1}^{2}-\lambda_{1}^{2}\right)-\left(b^{2}+b c\right)\left(X_{2}^{2}+Y_{2}^{2}-\lambda_{2}^{2}\right), \\
H_{2}:= & b c\left(X_{2}-1\right)^{2}+b c Y_{2}^{2},
\end{aligned}
$$

via elementary cancellation

$$
\gamma_{2}:=G_{2}+H_{2}=\left(b^{2}+b c\right) \lambda_{2}^{2}+c^{2}+b c-a^{2} \lambda_{1}^{2}
$$

and, symmetrically,

$$
\gamma_{3}:=\left(a^{2}+a c\right) \lambda_{1}^{2}+c^{2}+a c-b^{2} \lambda_{2}^{2} .
$$

Example 4.1. Let $a=1, b=2, c=5$. The curve (in $\lambda_{1}, \lambda_{2}$ ) given by (4.1) has a logarithmic image that is shown in Figure 3. Analogous special certificates can be obtained within the two other complement components.

Since (by homogenizing the polynomial) there is a symmetry, we obtain similarly an approximation of the other two complement components. Hence we have: 


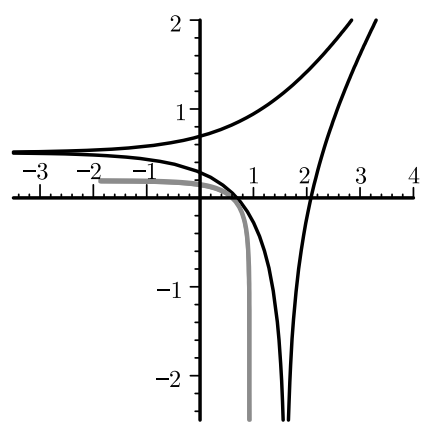

FiguRE 3. The boundary of an amoeba of a linear polynomial (black) and the logarithmic image of the boundary of $R_{1}=\{\lambda \in$ $\left.\mathbb{R}^{2}:\left(a^{2}+a b\right) \lambda_{1}^{2}+\left(b^{2}+a b\right) \lambda_{2}^{2}-c^{2}<0\right\}$, for which the special certificates of degree 2 exist (gray).

Lemma 4.2. Let $f=a Z_{1}+b Z_{2}+c$ with $a, b, c \in \mathbb{R}$, and set

$$
\begin{aligned}
& R_{1}=\left\{\lambda \in \mathbb{R}^{2}:\left(a^{2}+a b\right) \lambda_{1}^{2}+\left(b^{2}+a b\right) \lambda_{2}^{2}-c^{2}<0\right\}, \\
& R_{2}=\left\{\lambda \in \mathbb{R}^{2}:\left(b^{2}+b c\right) \lambda_{2}^{2}+c^{2}+b c-a^{2} \lambda_{1}^{2}<0\right\}, \\
& R_{3}=\left\{\lambda \in \mathbb{R}^{2}:\left(a^{2}+a c\right) \lambda_{1}^{2}+c^{2}+a c-b^{2} \lambda_{2}^{2}<0\right\} .
\end{aligned}
$$

Then $R_{j} \cap \mathcal{U}_{f}=\emptyset$ for $1 \leq j \leq 3$, and for any $\lambda \in R_{1} \cup R_{2} \cup R_{3}$ there exists a certificate (3.3) of degree at most two.

The monomial-based approach. For the monomial-based approach based on Corollary 3.7 we can provide special certificates for a much more general class. Our point of departure is Purbhoo's lopsidedness criterion [20] which guarantees that a point belongs to the complement of an amoeba $\mathcal{A}_{f}$. In particular, we can provide degree bounds for these certificates.

In the following let $\alpha(1), \ldots, \alpha(d) \in \mathbb{N}_{0}^{n}$ span $\mathbb{R}^{n}$ and $f=\sum_{j=1}^{d} b_{j} Z^{\alpha(j)} \in \mathbb{C}[Z]$ with monomials $m_{j}:=Z^{\alpha(j)}=Z_{1}^{\alpha(j)_{1}} \cdots Z_{n}^{\alpha(j)_{n}}$. For any point $v \in \mathbb{R}^{n}$ define $f\{v\}$ as the following sequence of numbers in $\mathbb{R}_{>0}$,

$$
f\{v\}:=\left(\left|b_{1} m_{1}\left(\log ^{-1}(v)\right)\right|, \ldots,\left|b_{d} m_{d}\left(\log ^{-1}(v)\right)\right|\right) .
$$

A list of positive real numbers is called lopsided if one of the numbers is greater than the sum of all the others. We call a point $v \in \mathbb{R}^{n}$ lopsided, if the sequence $f\{v\}$ is lopsided. Furthermore set

$$
\mathcal{L} \mathcal{A}(f):=\left\{v \in \mathbb{R}^{n}: f\{v\} \text { is not lopsided }\right\} .
$$

It is easy to see that $\mathcal{A}_{f} \subset \mathcal{L} \mathcal{A}(f)$ with $\mathcal{A}_{f} \neq \mathcal{L} \mathcal{A}(f)$ in general. In the following way the amoeba $\mathcal{A}_{f}$ can be approximated based on the lopsidedness concept. For $r \geq 1$ let

$$
\begin{aligned}
\tilde{f}_{r}(Z) & :=\prod_{k_{1}=0}^{r-1} \cdots \prod_{k_{n}=0}^{r-1} f\left(e^{2 \pi i k_{1} / r} Z_{1}, \ldots, e^{2 \pi i k_{n} / r} Z_{n}\right) \\
& =\operatorname{Res}\left(\operatorname{Res}\left(\ldots \operatorname{Res}\left(f\left(U_{1} Z_{1}, \ldots, U_{n} Z_{n}\right), U_{1}^{r}-1\right), \ldots, U_{n-1}^{r}-1\right), U_{n}^{r}-1\right),
\end{aligned}
$$

where Res denotes the resultant of two (univariate) polynomials. Then the following theorem holds. 
Theorem 4.3 (Purbhoo [20, Theorem 1]). (a) For $r \rightarrow \infty$ the family $\mathcal{L} \mathcal{A}\left(\tilde{f}_{r}\right)$ converges uniformly to $\mathcal{A}_{f}$. There exists an integer $N$ such that to compute $\mathcal{A}_{f}$ within $\varepsilon>0$, it suffices to compute $\mathcal{L} \mathcal{A}\left(\tilde{f}_{r}\right)$ for any $r \geq N$. Moreover, $N$ depends only on $\varepsilon$ and the Newton polytope (or degree) of $f$ and can be computed explicitly from these data.

(b) For an ideal $I \subset \mathbb{C}[Z]$ a point $v \in \mathbb{R}^{n}$ is in the amoeba $\mathcal{A}_{I}$ if and only if $g\{v\}$ is not lopsided for every $g \in I$.

Based on Theorem 4.3 one can devise a converging sequence of approximations for the amoeba. Note, however, that the lopsidedness criterion is not a Nullstellensatz in a strict sense since it does not provide a polynomial identity certifying membership in the complement of the amoeba.

The aim of this section is to figure out how our SOS approximation is related to the lopsidedness and transform the lopsidedness certificate into a certificate for the Nullstellensätze presented in Section 3 .

By Lemma 3.5 we can assume that the point $\lambda$, whose membership to an unlog amoeba $\mathcal{U}_{f}$ shall be decided, is the all-1-vector 1 . In this situation lopsidedness means that there is an index $j \in\{1, \ldots, d\}$ with $\left|b_{j}\right|>\sum_{i \neq j}\left|b_{i}\right|$. If the lopsidedness condition is satisfied in $v$, then the following statement provides a certificate of the form $G+H+1$ and bounded degree.

Corresponding to the definition of $I^{*}$ in (3.5), let the polynomials $s_{1}, \ldots, s_{d+2}$ be defined by

$$
s_{i}=\left(\frac{b_{i}^{\mathrm{re}}}{\left|b_{i}\right|} \cdot\left(Z^{\alpha(i)}\right)^{\mathrm{re}}\right)^{2}+\left(\frac{b_{i}^{\mathrm{im}}}{\left|b_{i}\right|} \cdot\left(Z^{\alpha(i)}\right)^{\mathrm{im}}\right)^{2}-1, \quad 1 \leq i \leq d,
$$

and $s_{d+1}=f^{\mathrm{re}}, s_{d+2}=f^{\mathrm{im}}$.

Theorem 4.4. If the point $\lambda=1$ is contained in the complement of $\mathcal{U}_{f}$ with $f\{0\}$ being lopsided with dominating element $\left|m_{1}(\mathbf{1})\right|$, then there exists a certificate of (total) degree $2 \cdot \operatorname{deg}(f)$ which is given by

$$
\sum_{i=1}^{d+2} s_{i} g_{i}+H+1=0,
$$

where

$$
\begin{aligned}
g_{1}= & \left|b_{1}\right|^{2}, \quad g_{i}=-\left|b_{i}\right| \cdot \sum_{k=2}^{d}\left|b_{k}\right|, \quad 2 \leq i \leq d, \\
g_{d+1}= & \left(-b_{1} \cdot Z^{\alpha(1)}+\sum_{i=2}^{d} b_{i} \cdot Z^{\alpha(i)}\right)^{\mathrm{re}}, \quad g_{d+2}=\left(-b_{1} \cdot Z^{\alpha(1)}+\sum_{i=2}^{d} b_{i} \cdot Z^{\alpha(i)}\right)^{\mathrm{im}}, \\
H= & \sum_{2 \leq i<j \leq d}\left|b_{i}\right| \cdot\left|b_{j}\right| \cdot\left(\frac{b_{i}^{\mathrm{re}}}{\left|b_{i}\right|} \cdot\left(Z^{\alpha(i)}\right)^{\mathrm{re}}-\frac{b_{j}^{\mathrm{re}}}{\left|b_{j}\right|} \cdot\left(Z^{\alpha(j)}\right)^{\mathrm{re}}\right)^{2} \\
& +\left|b_{i}\right| \cdot\left|b_{j}\right| \cdot\left(\frac{b_{i}^{\mathrm{im}}}{\left|b_{i}\right|} \cdot\left(Z^{\alpha(i)}\right)^{\mathrm{im}}-\frac{b_{j}^{\mathrm{im}}}{\left|b_{j}\right|} \cdot\left(Z^{\alpha(j)}\right)^{\mathrm{im}}\right)^{2} .
\end{aligned}
$$


Proof. By the third binomial formula $(\alpha+\beta) \cdot(\alpha-\beta)=\alpha^{2}-\beta^{2}$, substituting the polynomials $s_{i}$ and $g_{j}$ into $s_{d+1} g_{d+1}+s_{d+2} g_{d+2}$ yields

$-\left(b_{1}^{\mathrm{re}} \cdot\left(Z^{\alpha(1)}\right)^{\mathrm{re}}\right)^{2}+\left(\sum_{i=2}^{d}\left(b_{i} \cdot Z^{\alpha(i)}\right)^{\mathrm{re}}\right)^{2}-\left(b_{1}^{\mathrm{im}} \cdot\left(Z^{\alpha(1)}\right)^{\mathrm{im}}\right)^{2}+\left(\sum_{i=2}^{d}\left(b_{i} \cdot Z^{\alpha(i)}\right)^{\mathrm{im}}\right)^{2}$.

Adding $g_{1} s_{1}$ and the SOS term $H$ yields

$$
-\left|b_{1}\right|^{2}+\left(\sum_{j=2}^{d}\left(\frac{b_{j}^{\mathrm{re}}}{\left|b_{j}\right|} \cdot\left(Z^{\alpha(j)}\right)^{\mathrm{re}}\right)^{2}+\left(\frac{b_{j}^{\mathrm{im}}}{\left|b_{j}\right|} \cdot\left(Z^{\alpha(j)}\right)^{\mathrm{im}}\right)^{2}\right) \cdot\left(\left|b_{j}\right| \cdot \sum_{k=2}^{d}\left|b_{k}\right|\right) .
$$

Hence, the expression $\sum_{i=1}^{d+2} s_{i} g_{i}+H$ in (4.2) in total, results in

$$
-\left|b_{1}\right|^{2}+\left(\sum_{i=2}^{d}\left|b_{i}\right|\right)^{2}
$$

Since all $\left|b_{i}\right| \geq 0$ and by our assumption of lopsidedness with dominating term $\left|m_{1}(\mathbf{1})\right|$, this is the certificate we wanted to obtain. By rescaling, we can bring the constant to -1 .

We say that there exists a certificate for a point $w$ in the complement of the $(\log )$ amoeba $\mathcal{A}_{f}$ if there exists a certificate for the point $\mathbf{1}$ in the complement of the amoeba $\mathcal{U}_{g}$ in the sense of Theorem 4.4, where $g$ is defined as in Lemma 3.5 and $\lambda_{i}:=\left|\log ^{-1}\left(w_{i}\right)\right|$.

Corollary 4.5. Let $r \in \mathbb{N}$.

(1) For any $w \in \mathbb{R}^{n} \backslash \mathcal{L} \mathcal{A}\left(\tilde{f}_{r}\right) \subset \mathbb{R}^{n} \backslash \mathcal{A}_{f}$ there exists a certificate of degree at most $2 \cdot r^{n} \cdot \operatorname{deg}(f)$ which can be computed explicitly.

(2) The certificate determines the order of the complement component to which $w$ belongs.

Proof. By definition of $g$, we have $w \in \mathcal{A}_{f}$ if and only if $\mathbf{1} \in \mathcal{U}_{g}$. Further, $\mathbf{1}$ belongs to $\mathcal{L} \mathcal{A}\left(\tilde{g}_{r}\right)$ if and only if $\tilde{g}_{r}\{0\}$ is not lopsided. Applying Theorem 4.4 on the function $\tilde{g}_{r}$ yields a certificate for $w$ in the log amoeba $\mathcal{A}_{f}$. Since we have $\operatorname{tdeg}\left(\tilde{g}_{r}\right)=\operatorname{tdeg}(g) \cdot r^{n}=\operatorname{tdeg}(f) \cdot r^{n}$ due to the definition of $\tilde{g}_{r}$ and $g$, the result follows.

For the second statement, note that passing over from $f$ to $g$ does not change the order $\nu$ of any point in the complement of the amoebas. Now it suffices to show that the dominating term (which occurs in a distinguished way in the certificate) determines the order of the complement component. The latter statement follows from Purbhoo's result that if $w \notin \mathcal{L A}\left(\hat{f}_{r}\right)$ and the order of the complement component of $w$ is $\alpha(i)$, then the dominant term in $\tilde{f}_{r}$ has the exponent $r^{n} \cdot \alpha(i)$ (see [20, Proposition 4.1]).

Corollary 4.6. For linear hyperplane amoebas in $\mathbb{R}^{n}$, any point in the complement of the amoeba has a certificate whose sum of squares is a sum of squares of affine functions.

Proof. By the explicit characterization of linear hyperplane amoebas in 2, Corollary 4.3], any point in the complement is lopsided. Hence, the statement follows from Theorem 4.4 . 
Simplified expressions. From a slightly more general point of view, the monomial-based certificates can be seen as a special case of the following construction. Whenever the defining polynomials of a variety originate from simpler polynomials with algebraically independent monomials, then the approximation of the amoeba can be simplified.

For an ideal $I$ let $V:=\mathcal{V}(I) \subset\left(\mathbb{C}^{*}\right)^{n}$ be its subvariety in $\left(\mathbb{C}^{*}\right)^{n}$. Let $\gamma_{1}, \ldots, \gamma_{k}$ be $k$ monomials in $n$ variables, say, $\gamma_{i}=Y^{\alpha(i)}=Y_{1}^{\alpha(i)_{1}} Y_{2}^{\alpha(i)_{2}} \cdots Y_{n}^{\alpha(i)_{n}}$, where $\alpha(i)=\left(\alpha(i)_{1}, \ldots, \alpha(i)_{n}\right) \in \mathbb{Z}^{n}$. They define a homomorphism $\gamma$ of algebraic groups from $\left(\mathbb{C}^{*}\right)^{n}$ to $\left(\mathbb{C}^{*}\right)^{k}$. For any subvariety $W$ of $\left(\mathbb{C}^{*}\right)^{k}$, the inverse image $\gamma^{-1}(W)$ is a subvariety of $\left(\mathbb{C}^{*}\right)^{n}$. Note that the map $\gamma$ is onto if and only if the vectors $\alpha(1), \ldots, \alpha(k)$ are linearly independent (see [26, Lemma 4.1]).

Let $J$ be an ideal with $\mathcal{V}(J)=\gamma^{-1}(V)$. If the map $\gamma$ is onto, then computing the amoeba of $J$ can be reduced to the computation of the amoeba of $I$. Let $\gamma^{\prime}$ denote the restriction of $\gamma$ to the multiplicative subgroup $(0, \infty)^{n}$. Then the following diagram is a commutative diagram of multiplicative abelian groups:

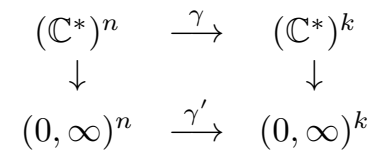

where the vertical maps are taking coordinatewise absolute value. For vectors $p=\left(p_{1}, \ldots, p_{n}\right)$ in $\left(\mathbb{C}^{*}\right)^{n}$ we write $|p|=\left(\left|p_{1}\right|, \ldots,\left|p_{n}\right|\right) \in(0, \infty)^{n}$, and similarly for vectors of length $k$. Further, for $V \subset\left(\mathbb{C}^{*}\right)^{n}$ let $|V|:=\{|p|: p \in V\}$. If the map $\gamma$ is onto, then $\left|\gamma^{-1}(V)\right|=\gamma^{-1}(|V|)$ (see [26]).

Theorem 4.7. If a point outside of an unlog amoeba $\mathcal{U}_{I}$ has a certificate of total degree $d$, then a point outside of the unlog $\mathcal{U}_{J}$ has a certificate of degree $d \cdot D$, where $D$ is the maximal total degree of the monomials $\gamma_{1}, \ldots, \gamma_{k}$.

In particular, this statement applies to the certificates from Corollaries 3.2 and 3.7.

Proof. Let $p$ be a point outside of the unlog amoeba of $V \subset\left(\mathbb{C}^{*}\right)^{n}$ which has a certificate of total degree $d$. By Corollary 3.2 , the certificate consists of a polynomial $G(X, Y)$ in the real ideal $I^{\prime} \subset \mathbb{R}[X, Y]$ from (3.1) and by real sums of squares of polynomials in $\mathbb{R}[X, Y]$. For the polynomials in the ideals, we observe that the realification process carries over to the substitution process. W.l.o.g. we can assume that $\gamma_{i}$ is a product of just two factors. Then, with $Z=Z_{1}+i Z_{2}, Z=P Q$ we have $Z_{1}+i Z_{2}=\left(P_{1}+i P_{2}\right)\left(Q_{1}+i Q_{2}\right)$ and use the real substitutions $Z_{1} \equiv P_{1} Q_{1}-P_{2} Q_{2}$, $Z_{2} \equiv P_{1} Q_{2}+P_{2} Q_{1}$, and in the same way the real sums of squares remain real sums of squares (of the polynomials in $P_{i}, Q_{j}$ ) after substituting.

Example 4.8. Let $\mathbb{G}_{1,3}$ denote the Grassmannian of lines in 3-space, which is the variety in $\mathbb{P}_{\mathbb{C}}^{5}$, defined by

$$
P_{01} P_{23}-P_{02} P_{13}+P_{03} P_{12}=0
$$

which we consider as a subvariety of $\left(\mathbb{C}^{*}\right)^{6}$. The three terms in this quadratic equation involve distinct variables and hence correspond to linearly independent exponent vectors. Note that $\mathbb{G}_{1,3}$ equals $\gamma^{-1}(V)$ where

$$
\gamma:\left(\mathbb{C}^{*}\right)^{6} \rightarrow\left(\mathbb{C}^{*}\right)^{3},\left(p_{01}, p_{02}, p_{03}, p_{12}, p_{13}, p_{23}\right) \mapsto\left(p_{01} p_{23}, p_{02} p_{13}, p_{03} p_{12}\right)
$$




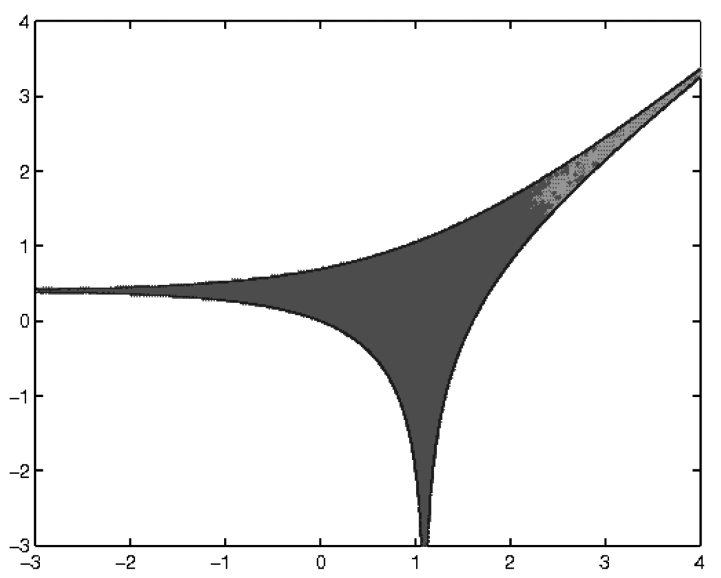

FigURE 4. SOS certificates of linear amoebas restricted to degree two. The dark gray points represent infeasible SDPs, and in the light gray points numerical instabilities were reported in the computations.

and $V$ denotes the plane in 3-space defined by the linear equation $X-Y+Z=0$. Since by Corollary 4.6 any point in the complement has a certificate of degree 2, Theorem 4.7 implies that every point in the complement of the Grassmannian amoeba has a certificate of degree 4 .

\section{Computing the Relaxations Via SEmidefinite PROGRAmming}

We close this paper by providing some computational results in order to confirm the validity of our approach. The subsequent computations have been performed on top of SOSTOOLS [18 which is a MATLAB package for computing sums of squares based computations. The SDP package underlying SOSTOols is SEDUMi [23].

Example 5.1. For the test case of a linear polynomial $f:=Z_{1}+2 Z_{2}+3$, the boundary contour of the amoeba $\mathcal{A}_{f}$ can be explicitly described, and it is given by the curves

$$
\begin{aligned}
\exp \left(z_{1}\right) & =2 \cdot \exp \left(z_{2}\right)+3 \\
2 \cdot \exp \left(z_{2}\right) & =\exp \left(z_{1}\right)+3 \\
3 & =\exp \left(z_{1}\right)+2 \cdot \exp \left(z_{2}\right) ;
\end{aligned}
$$

see [2. We compute the amoeba of $f$ with our SDP via SOSTOoLs on a grid of size $250 \times 250$ lattice points in the area $[-3,4]^{2}$. In the SDP we restrict to polynomials of degree two. By Theorem 4.4 the approximation is exact in that case (up to numerical issues). Figure 4 visualizes the SDP-based computation of the SOS certificates. In the figure, at the white outer regions, certificates are found. At the dark gray points the SDP is infeasible; at the light gray points, no feasibility is proven, but numerical instabilities are reported by the SDP solver. The aspect of numerical stability of our SOS-based amoeba computations and of general SOS computations is an important issue in convex algebraic geometry which deserves 
further study. See [7, 25] for existing work in this direction, based on choosing different bases.

Example 5.2. As in Figure 1 we consider the class of polynomials $f:=Z_{1}^{2} Z_{2}+$ $Z_{1} Z_{2}^{2}+c \cdot Z_{1} Z_{2}+1$ with some constant $c \in \mathbb{R}$. We use the monomial-based approach from Corollary 3.7. In order to compute whether a given point $\left(\lambda_{1}, \lambda_{2}\right) \in(0, \infty)^{2}$ is contained in the unlog amoeba $\mathcal{U}_{f}$, we have to consider the polynomials

$$
\begin{aligned}
h_{1}= & \left(X_{1}^{2} X_{2}-2 X_{1} Y_{1} Y_{2}-Y_{1}^{2} \cdot X_{2}\right)+\left(X_{1} X_{2}^{2}-X_{1} Y_{2}^{2}-2 Y_{1} X_{2} Y_{2}\right) \\
& +c \cdot\left(X_{1} X_{2}-Y_{1} Y_{2}\right)+1, \\
h_{2}= & \left(X_{1}^{2} Y_{2}+2 X_{1} Y_{1} X_{2}-Y_{1}^{2} Y_{2}\right)+\left(2 X_{1} X_{2} Y_{2}+Y_{1} X_{2}^{2}-Y_{1} Y_{2}^{2}\right)+c \cdot\left(X_{1} Y_{2}+X_{2} Y_{1}\right), \\
h_{3}= & \left(X_{1}^{2} X_{2}-2 X_{1} Y_{1} Y_{2}-Y_{1}^{2} X_{2}\right)^{2}+\left(X_{1}^{2} Y_{2}+2 X_{1} Y_{1} X_{2}-Y_{1}^{2} Y_{2}\right)^{2}-\left(\lambda_{1}^{2} \cdot \lambda_{2}\right)^{2}, \\
h_{4}= & \left(X_{1} X_{2}^{2}-X_{1} Y_{2}^{2}-2 Y_{1} X_{2} Y_{2}\right)^{2}+\left(2 X_{1} X_{2} Y_{2}+Y_{1} X_{2}^{2}-Y_{1} Y_{2}^{2}\right)^{2}-\left(\lambda_{1} \cdot \lambda_{2}^{2}\right)^{2}, \\
h_{5}= & \left(X_{1} X_{2}-Y_{1} Y_{2}\right)^{2}+\left(X_{1} Y_{2}+X_{2} Y_{1}\right)^{2}-\left(\lambda_{1} \cdot \lambda_{2}\right)^{2} .
\end{aligned}
$$

For the case $c=2$ and $c=-4$ we investigate $160 \times 160$ points in the area $[-4,4] \times[-5,3]$ and restrict the polynomials multiplied with the constraints to degree three, and thus restrict ourselves to a degree bound of six. The resulting amoeba $\mathcal{A}_{f}$ is depicted in Figure 5. At the white points the SDP is feasible and thus these points belong to the complement component. At the light gray points the SDP is recognized as feasible with numerical issues (within a pre-defined range). At the black points in the center the SDP was infeasible without numerical issues and at the dark gray points with numerical issues reported. At the black points in the upper right corner the program stopped due to exceeding numerical problems. The union of the central black, the dark gray and part of the light gray points provides the (degree bounded) approximation of the amoeba.
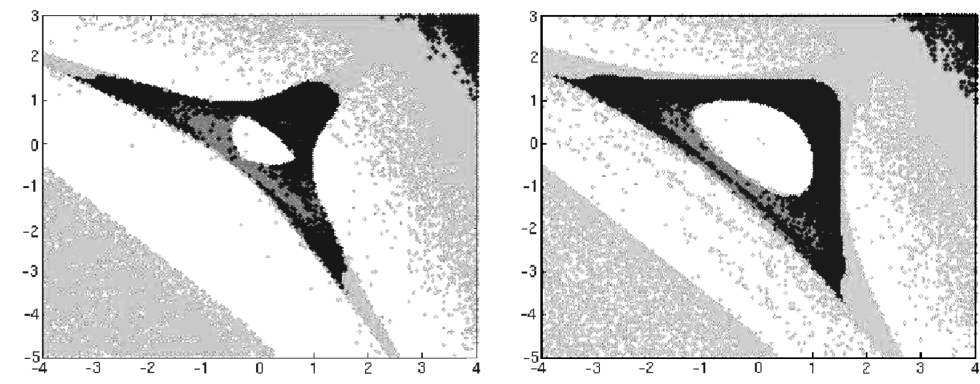

Figure 5. The amoeba of $f:=Z_{1}^{2} Z_{2}+Z_{1} Z_{2}^{2}+c \cdot Z_{1} Z_{2}+1$ approximated with SOSTOOLS for $c=2$ and $c=-4$. See Example 5.2 for an explanation of the grayscales and an address of the numerical issues.

The diameter of inner complement components. We briefly discuss that the SOS-based certificates can also be used for more sophisticated questions rather than the pure membership problem. For this, we consider a family of polynomials $f \in \mathbb{C}\left[Z_{1}, \ldots, Z_{n}\right]$ whose Newton polytope is a simplex and which have $n+2$ monomials such that one of them is located in the interior of the simplex. Amoebas 
of polynomials of this class have at most one inner complement component (see [27] for a comprehensive investigation of that class).

Let $f:=\sum_{i=0}^{n+1} b_{i} \cdot Z^{\alpha(i)}$, and let $b_{0}$ denote the coefficient of the inner monomial. By the results in [27], for $\left|b_{0}\right| \rightarrow \infty$ the inner complement component appears at the image under the Log-map of a minimal point $\delta$ of the function $\hat{f}:=\left|\frac{f}{\arg \left(b_{0}\right) \cdot Z^{\alpha(0)}}\right|$. $\delta$ is explicitly computable, and $|\delta|$ is unique. This allows us to certify that a complement component of the unlog amoeba has a certain diameter $d$ under the scaling $|Z| \mapsto|Z|^{2}$ of the (unlog) amoeba basis space by solving the SDP corresponding to

$$
\sum_{j=1}^{3} s_{j} g_{j}+H+1=0
$$

with $s_{1}=\sum_{i=1}^{n}\left(\left|\delta_{i}\right|^{2}-\left|Z_{i}\right|^{2}\right)^{2}-d^{2} / 4, s_{2}=\sum_{i=0}^{n+1}\left(b_{i} \cdot Z^{\alpha(i)}\right)^{\mathrm{re}}$ and $s_{3}=\sum_{i=0}^{n+1}$ $\left(b_{i} \cdot Z^{\alpha(i)}\right)^{\mathrm{im}}$, where $g_{j} \in \mathbb{C}[Z]$ (restricted to some total degree) and $H$ is an SOS polynomial.

Feasibility of the SDP certifies that there exists no point $v \in \mathcal{V}(f) \cap \partial \mathcal{B}_{d / 2}(\delta)$ (where $\mathcal{B}_{d / 2}(\delta)$ denotes the ball with radius $d / 2$ centered in $\delta$ ) in the rescaled amoeba basis space. Hence, the corresponding inner complement component of the unlog amoeba has at least a diameter $d$ in that space. We have to investigate the rescaled basis space of the unlog amoeba in order to transform the generic condition $\left(\left|\delta_{i}\right|-\left|Z_{i}\right|\right)^{2}-d^{2} / 4$ on the basis space of $\mathcal{U}_{f}$ into a polynomial condition, which is given by $s_{1}$ here.

Note that this works not only for polynomials in the class under investigation, but for every polynomial as long as one knows, where a complement component appears.

Example 5.3. As before, let $f:=Z_{1}^{2} Z_{2}+Z_{1} Z_{2}^{2}+c \cdot Z_{1} Z_{2}+1$ with a real parameter $c$. For this class, the inner complement component appears at the point $(1,1)$ and thus under the Log-map at the origin of $\log \left(\mathbb{R}^{2}\right)$. The inner complement component exists for $c>1$ and $c<-3$ (cf. [27]). We compute a bound for the diameter of the inner complement component using the upper SDP for the intervals $[-3,-9]$ and $[1,7]$ with steplength 0.1 . For any of these points we compute 14 SDPs in order to estimate the radius (based on binary search). In the rescaled amoeba basis space we obtain the bounds shown in Figure 6 .

Observe that these bounds are lower bounds since feasibility of the SDP certifies membership in the complement of the amoeba but infeasibility only certifies that no certificate with polynomials of degree at most $k$ (i.e., 3 in our case) exists.

This approach also yields lower bounds for the diameter of the inner complement component of the (log) amoeba. The image of the circle $\sum_{i=1}^{n}\left(\left|\delta_{i}\right|^{2}-\left|Z_{i}\right|^{2}\right)^{2}-r^{2}$ under rescaling and the $\log -$ map, i.e., $\sum_{i=1}^{n}\left(\log \left|\delta_{i}\right|-\log \left|Z_{i}\right|\right)^{2}-r^{2}$, contains the set of points

$$
\left\{\left(\left|\delta_{1}\right| \cdot e^{r}, \ldots,\left|\delta_{n}\right|\right), \ldots,\left(\left|\delta_{1}\right|, \ldots,\left|\delta_{n}\right| \cdot e^{r}\right),\left(\left|\delta_{1}\right| \cdot e^{\frac{r}{\sqrt{n}}}, \ldots,\left|\delta_{n}\right| \cdot e^{\frac{r}{\sqrt{n}}}\right)\right\} .
$$

By convexity of the complement components in $\mathcal{A}_{f}$, the simplex spanned by these points is contained in the inner complement component. Hence the double radius of the insphere of that simplex is a lower bound for the diameter of the inner complement component of $\mathcal{A}_{f}$. 

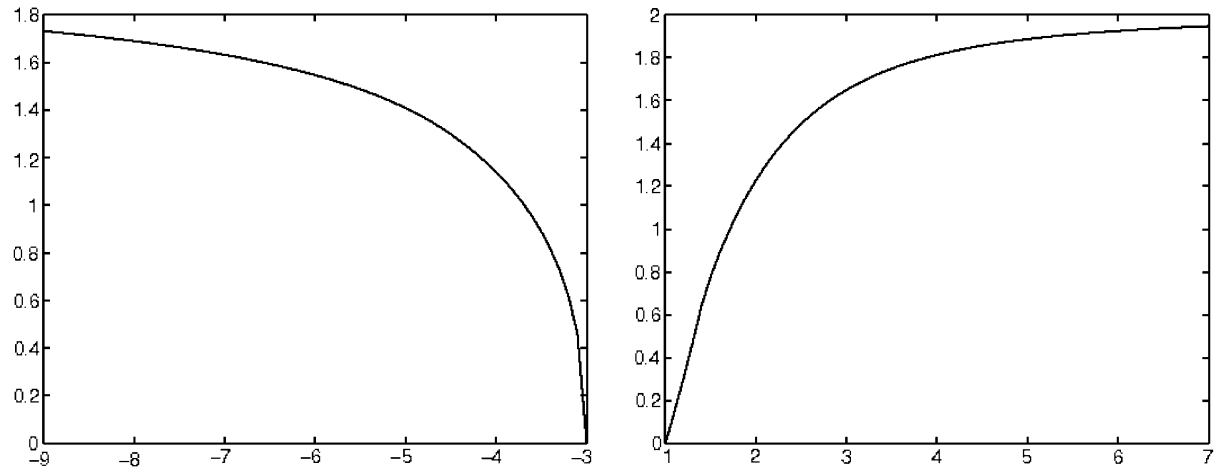

Figure 6. Lower bound for the diameter of the inner complement component.

\section{OutLOOK}

We have developed foundations and techniques for approximating amoebas and coamoebas based on the real Nullstellensatz and sums of squares techniques. While our focus was on developing the core principles of the computational methodology, some experimental results were provided to show the validity of the approach. Beyond the specific results we have presented, our approach can be seen as a first systematic treatment of amoebas from a (computational) real algebraic point of view and we think that this viewpoint will have more potential to offer.

Major current challenges involve both computational issues (concerning the quality and efficiency of computations) as well theoretical questions on the real algebraic viewpoint on amoebas. To name a specific open question in the latter respect, recall that in Theorem 4.4 for a hypersurface amoeba $\mathcal{A}_{f}$ we could deduce the order of a complement component from the special certificates we treated in that theorem. However, it is an open and important structural question how to deduce the order for a point $w$ in the complement of $\mathcal{A}_{f}$ given an arbitrary Nullstellensatz certificate for $w$.

\section{ACKNOWLEDGMENTS}

The authors thank Mihai Putinar for pointing out reference [10] and an anonymous referee for careful reading and helpful suggestions. Part of this work was done while the first author was visiting Universidad de Cantabria in Santander. He would like to express his gratitude for the hospitality.

\section{REFERENCES}

[1] Jacek Bochnak, Michel Coste, and Marie-Françoise Roy, Real algebraic geometry, Ergebnisse der Mathematik und ihrer Grenzgebiete (3) [Results in Mathematics and Related Areas (3)], vol. 36, Springer-Verlag, Berlin, 1998. Translated from the 1987 French original; Revised by the authors. MR 1659509 (2000a:14067)

[2] Mikael Forsberg, Mikael Passare, and August Tsikh, Laurent determinants and arrangements of hyperplane amoebas, Adv. Math. 151 (2000), no. 1, 45-70, DOI 10.1006/aima.1999.1856. MR:1752241 (2001m:32060)

[3] I. M. Gel'fand, M. M. Kapranov, and A. V. Zelevinsky, Discriminants, resultants, and multidimensional determinants, Mathematics: Theory \& Applications, Birkhäuser Boston Inc., Boston, MA, 1994. MR 1264417 (95e:14045) 
[4] Jean B. Lasserre, Global optimization with polynomials and the problem of moments, SIAM J. Optim. 11 (2000/01), no. 3, 796-817, DOI 10.1137/S1052623400366802. MR1814045 (2002b:90054)

[5] Monique Laurent, Sums of squares, moment matrices and optimization over polynomials, Emerging applications of algebraic geometry, IMA Vol. Math. Appl., vol. 149, Springer, New York, 2009, pp. 157-270, DOI 10.1007/978-0-387-09686-5_7. MR2500468 (2010j:13054)

[6] J.A. De Loera, J. Lee, P.N. Malkin, and S. Margulies, Hilbert's Nullstellensatz and an algorithm for proving combinatorial infeasibility, Proc. ISSAC 2008, ACM, New York, 2008, pp. 197-206.

[7] J. Löfberg and P. Parrilo, From coefficients to samples: a new approach to SOS optimization, 43rd IEEE Conference on Decision and Control, Atlantis, vol. 3, 2004, pp. 3154-3159.

[8] D. Maclagan and B. Sturmfels, Introduction to Tropical Geometry, Manuscript, 2010.

[9] Grigory Mikhalkin, Amoebas of algebraic varieties and tropical geometry, Different faces of geometry, Int. Math. Ser. (N. Y.), vol. 3, Kluwer/Plenum, New York, 2004, pp. 257-300, DOI 10.1007/0-306-48658-X_6. MR.2102998 (2005m:14110)

[10] Silviu-Iulian Niculescu and Mihai Putinar, A toric Positivstellensatz with applications to delay systems (English, with English and French summaries), C. R. Math. Acad. Sci. Paris 349 (2011), no. 5-6, 327-329, DOI 10.1016/j.crma.2010.11.018. MR2783329

[11] L. Nilsson, Amoebas, discriminants, and hypergeometric functions, Ph.D. thesis, Stockholm University, 2009.

[12] Lisa Nilsson and Mikael Passare, Discriminant coamoebas in dimension two, J. Commut. Algebra 2 (2010), no. 4, 447-471, DOI 10.1216/JCA-2010-2-4-447. MR 2753718(2011k:14033)

[13] Lisa Nilsson and Mikael Passare, Mellin transforms of multivariate rational functions, J. Geom. Anal. 23 (2013), no. 1, 24-46, DOI 10.1007/s12220-011-9235-7. MR3010271

[14] M. Nisse, Geometric and combinatorial structure of hypersurface coamoebas, Preprint, arXiv:0906.2729, 2009.

[15] Pablo A. Parrilo, Semidefinite programming relaxations for semialgebraic problems, Math. Program. 96 (2003), no. 2, Ser. B, 293-320, DOI 10.1007/s10107-003-0387-5. Algebraic and geometric methods in discrete optimization. MR.1993050 (2004g:90075)

[16] Pablo A. Parrilo, Exploiting algebraic structure in sum of squares programs, Positive polynomials in control, Lecture Notes in Control and Inform. Sci., vol. 312, Springer, Berlin, 2005, pp. 181-194. MR.2123524 (2005i:93038)

[17] Mikael Passare and August Tsikh, Amoebas: their spines and their contours, Idempotent mathematics and mathematical physics, Contemp. Math., vol. 377, Amer. Math. Soc., Providence, RI, 2005, pp. 275-288, DOI 10.1090/conm/377/06997. MR2149010(2006d:32005)

[18] Stephen Prajna, Antonis Papachristodoulou, Peter Seiler, and Pablo A. Parrilo, SOSTOOLS and its control applications, Positive polynomials in control, Lecture Notes in Control and Inform. Sci., vol. 312, Springer, Berlin, 2005, pp. 273-292. MR.2123527 (2005j:93004)

[19] Alexander Prestel and Charles N. Delzell, Positive polynomials, Springer Monographs in Mathematics, Springer-Verlag, Berlin, 2001. From Hilbert's 17th problem to real algebra. MR1829790 (2002k:13044)

[20] Kevin Purbhoo, A Nullstellensatz for amoebas, Duke Math. J. 141 (2008), no. 3, 407-445, DOI 10.1215/00127094-2007-001. MR2387427(2009b:14114)

[21] Mihai Putinar, Positive polynomials on compact semi-algebraic sets, Indiana Univ. Math. J. 42 (1993), no. 3, 969-984, DOI 10.1512/iumj.1993.42.42045. MR1254128 (95h:47014)

[22] Jürgen Richter-Gebert, Bernd Sturmfels, and Thorsten Theobald, First steps in tropical geometry, Idempotent mathematics and mathematical physics, Contemp. Math., vol. 377, Amer. Math. Soc., Providence, RI, 2005, pp. 289-317, DOI 10.1090/conm/377/06998. MR2149011 (2006d:14073)

[23] Jos F. Sturm, Using SeDuMi 1.02, a MATLAB toolbox for optimization over symmetric cones, Optim. Methods Softw. 11/12 (1999), no. 1-4, 625-653, DOI 10.1080/10556789908805766. Interior point methods. MR 1778433

[24] Bernd Sturmfels, Solving systems of polynomial equations, CBMS Regional Conference Series in Mathematics, vol. 97, Published for the Conference Board of the Mathematical Sciences, Washington, DC, 2002. MR.1925796 (2003i:13037)

[25] J.L. Sun, Low rank decompositions for sum of squares optimization, M.Sc. thesis, M.I.T., 2006. 
[26] Thorsten Theobald, Computing amoebas, Experiment. Math. 11 (2002), no. 4, 513-526 (2003). MR 1969643 (2004b:14100)

[27] Thorsten Theobald and Timo de Wolff, Amoebas of genus at most one, Adv. Math. 239 (2013), 190-213, DOI 10.1016/j.aim.2013.03.001. MR3045147

[28] Henry Wolkowicz, Romesh Saigal, and Lieven Vandenberghe (eds.), Handbook of semidefinite programming, International Series in Operations Research \& Management Science, 27, Kluwer Academic Publishers, Boston, MA, 2000. Theory, algorithms, and applications. MR,1778223 (2001k:90001)

Goethe-Universität, FB 12 - Institut für Mathematik, Postfach 1119 32, D-60054 Frankfurt am Main, Germany

E-mail address: theobald@math.uni-frankfurt.de

Goethe-Universität, FB 12 - Institut für Mathematik, Postfach 1119 32, D-60054 Frankfurt am Main, Germany

Current address: Universität des Saarlandes, Fachrichtung Mathematik, Postfach 151150, 66041 Searbrücken, Germany

E-mail address: dewolff@math.uni-sb.de 